LHCb 2004-005, RICH

February 26, 2004

\title{
Study of ageing effects in aerogel
}

\author{
T. Bellunato ${ }^{1}$, M. Calvi ${ }^{1}$, C. Coluzza ${ }^{2}$, G. Longo ${ }^{2}$, \\ C. Matteuzzi ${ }^{1}$, M. Musy ${ }^{1}$, P. Negri $^{1}$, D. L. Perego ${ }^{1}$ \\ ${ }^{1}$ Università degli Studi di Milano Bicocca and INFN, Milano, Italy; \\ ${ }^{2}$ INFM and Università di Roma "La Sapienza", Roma, Italy
}

\begin{abstract}
Ageing effects on aerogel due to irradiation and absorption of humidity have been investigated. Aerogel tiles have been exposed to $\gamma$ radiation from a ${ }^{60} \mathrm{Co}$ source and to proton and neutron high intensity beams. The transmittance has been monitored in the wavelength range between $200 \mathrm{~nm}$ and $800 \mathrm{~nm}$, determining the clarity factor $C$ as a function of the increasing dose of irradiation. The index of refraction $n$ was also measured.
\end{abstract}




\section{Introduction}

Silica aerogel is a solid material made of $\mathrm{SiO}_{2}[1,2,3]$ with a very low density ranging between about 0.003 and $0.35 \mathrm{~g} / \mathrm{cm}^{3}$. It is transparent and its refractive index can be tuned within the wide range of 1.008 - 1.08. Depending on the manufacturing procedure, the final product can be hygroscopic or hydrophobic. The use of aerogel as radiator for RICH detectors [4] demands extremely good optical quality [5, 6, 7]. This has now been achieved [8], and several experiments have built Ring Imaging CHerenkov detectors (RICH) with aerogel, like HERMES [9] or plan to build one, like LHCb [10], AMS [11] and BELLE [12].

Possible ageing of aerogel due to intense irradiation from different sources or absorption of humidity has been studied. Aerogel tiles were irradiated with very intense $\gamma$, proton and neutron beams or exposed to humid air. Optical parameters like transmittance, clarity and index of refraction were measured to monitor ageing. The hygroscopic aerogel used has been produced by the Boreskov Institute of Catalysis in Novosibirsk [2]. The tiles used in the tests presented in this paper have dimensions of about $50 \times 50 \times 20 \mathrm{~mm}^{3}$ and the index of refraction $n$ is around 1.03 .

\section{Optical Properties of Aerogel}

The light diffusion within the aerogel is usually the factor limiting the performance of this material as Cherenkov radiator for a RICH detector. The dominant contribution to the total diffusion probability comes from the Rayleigh scattering mechanism. The scattering cross section for a photon of wavelength $\lambda$ is proportional to $\lambda^{-4}$.

The absorption cross section is, within a wide range, wavelength independent and can be neglected when considering samples a few centimetres thick. The transmittance $T$ of the aerogel can be parametrized by the Hunt formula [13]:

$$
T(\lambda)=\frac{I}{I_{0}}=A e^{-C \cdot t / \lambda^{4}}
$$

where $I_{0}$ and $I$ are the incident and the transmitted intensities of the light beam through the sample. $A$ is the surface scattering coefficient, $t$ is the thickness of the aerogel block, and $\lambda$ is the wavelength of the impinging light beam. $C$ is the clarity factor and it is used to specify the optical quality of a sample together with $A$. An ideal aerogel would have $A$ and $C$ close to 1 and 0 respectively. Ageing effects have been monitored measuring the transmittance $T$ and extracting the parameters $A$ and $C$ at several intermediate steps during irradiation and the exposure to humidity.

\subsection{Transmittance Measurements}

The transmittance $T$ was measured by means of a double beam spectrophotometer. A scan in the wavelength range between $200 \mathrm{~nm}$ and $800 \mathrm{~nm}$ was performed in steps of 1 $\mathrm{nm}$.

An example of a measured spectrum is shown in Fig. 1 for a thickness of $21 \mathrm{~mm}$. The curve is fitted with the Hunt formula (1) in order to determine the parameters $A$ and $C$. The correlation coefficient between these two parameters is about 0.7. 


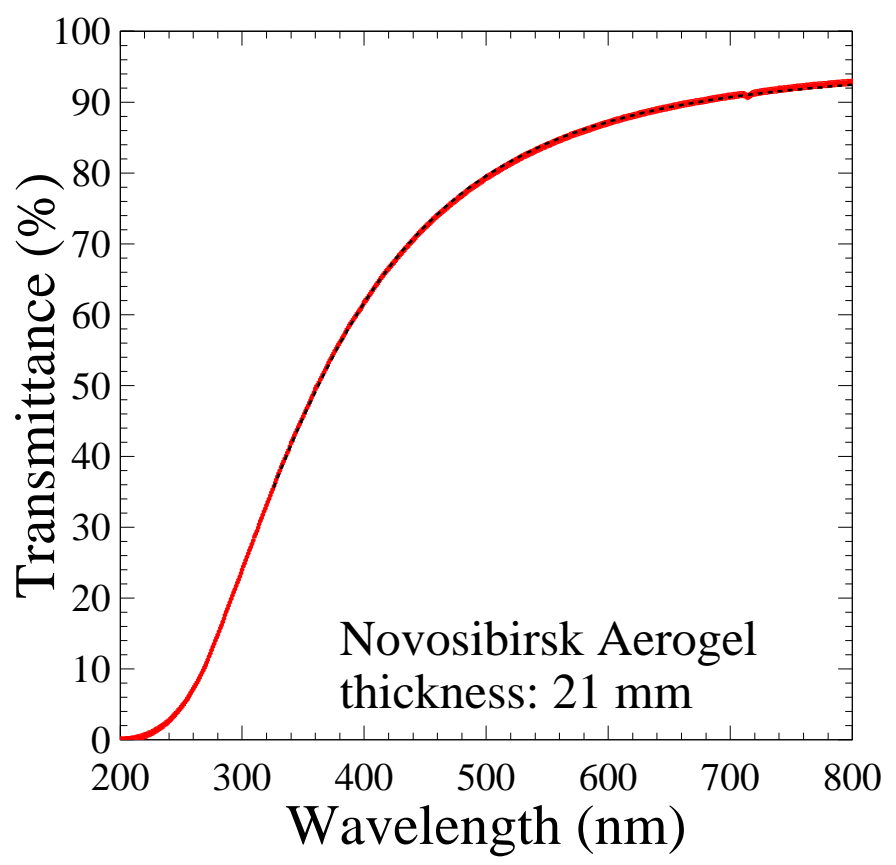

Figure 1: An example of a transmittance measurement fitted with the Hunt relation (1). The result of the fit, shown as the black dotted line, is hardly visible because superimposed to the measurement. It gives $A=(94.97 \pm 0.04) \%$ and $C=(0.00526 \pm 0.00001) \mu \mathrm{m}^{4} / \mathrm{cm}$.

To estimate the point to point variation of the Hunt parameters $A$ and $C$, several measurements have been performed with the spectrophotometer beam entrance point scanning the two large $\left(50 \times 50 \mathrm{~mm}^{2}\right)$ surfaces. The dispersion observed in these measurements was assumed to be a systematic error on $A$ and $C$ for the whole tile. As shown in Fig. 2 (a), for hygroscopic aerogel the transmittance at $400 \mathrm{~nm}$ is uniform in the tile at the level of $3-4 \%$. The dispersion of the fitted value of the clarity $C$ is about $2 \%$, as shown in Fig. 2 (b). In the case of hydrophobic aerogel, these dispersions are about $1-2 \%$ for the transmittance at $400 \mathrm{~nm}$ and $7 \%$ for the clarity $C$ respectively.

\subsection{Measuring the Index of Refraction}

The refractive index $n$ of the aerogel was measured using green $(\lambda=543.5 \mathrm{~nm})$ and red $(\lambda=632.8 \mathrm{~nm}) \mathrm{He}-\mathrm{Ne}$ laser sources. The measurements were performed using the prism method. Fig. 3 shows the block positioned in front of the laser beam on a rotating table. The sample is rotated until the deflection angle $\theta_{\text {out }}$ reaches its minimum. In that condition the index of refraction is given by:

$$
n=\sin \left(\frac{\Phi+\theta_{\text {out }}}{2}\right) / \sin \left(\frac{\Phi}{2}\right)
$$


a)

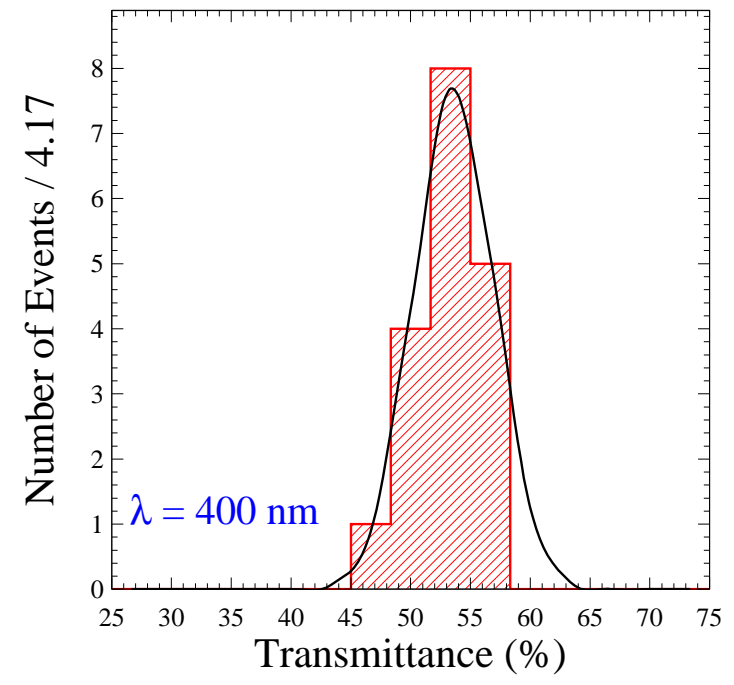

b)

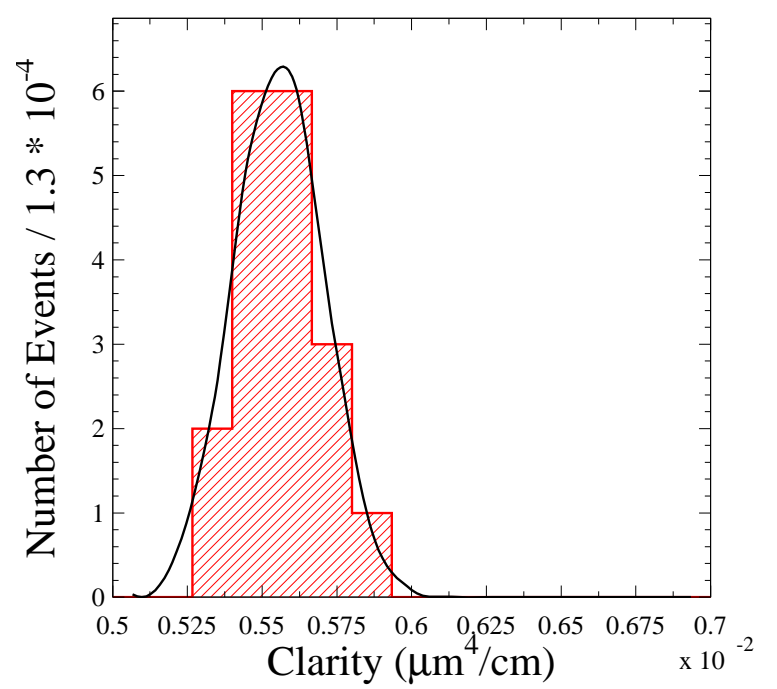

Figure 2: Distributions for a) transmittance $T$ at $\lambda=400 \mathrm{~nm}$ and b) clarity factor $C$. A Gaussian fit is superimposed, yielding $\sigma=3.3 \%$ for the transmittance at $400 \mathrm{~nm}$ and $\sigma=0.13 \cdot 10^{-3} \mu \mathrm{m}^{4} / \mathrm{cm}$ for the clarity factor.

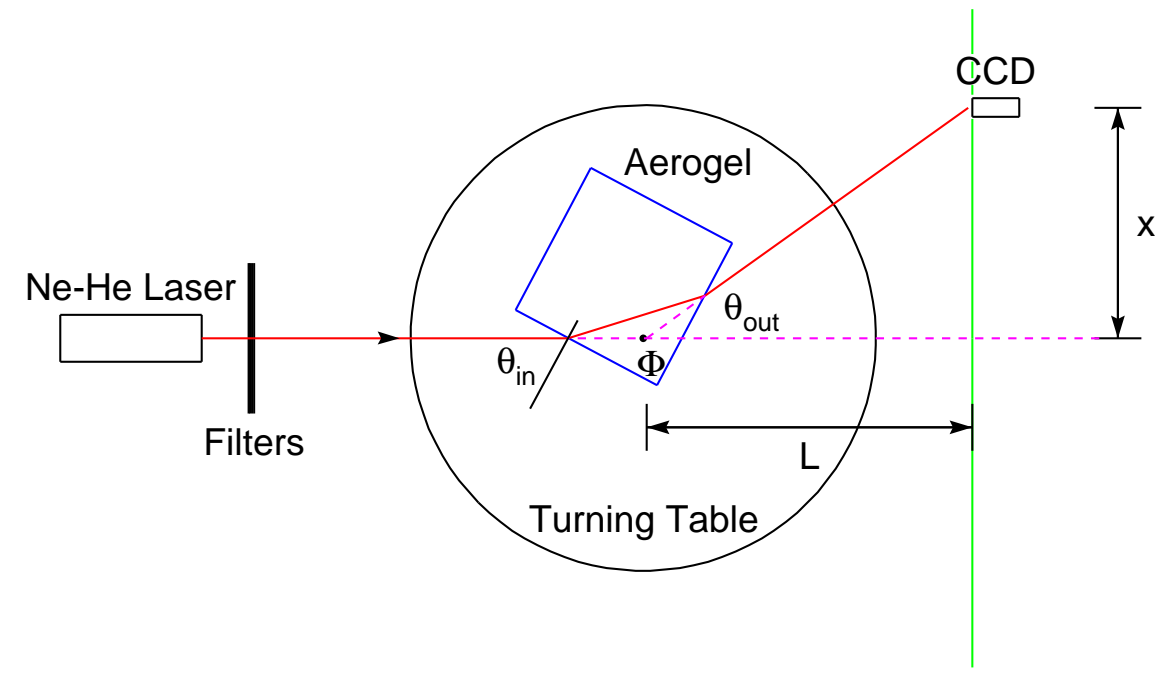

Figure 3: Experimental set-up used for the measurement of the refraction index.

where $\Phi$ is the angle between the two adjacent sides of the block $\left(\Phi \simeq 90^{\circ}\right) ; \theta_{\text {out }}$, the deflection angle, is determined by measuring the displacement $x$ with a CCD camera ${ }^{1}$ located at $L=870 \mathrm{~mm}$ from the aerogel.

\footnotetext{
${ }^{1} \mathrm{SONY} \circledast \mathrm{XC}-\mathrm{ST} 70 \mathrm{CE}$ with $768 \times 512$ pixels and a sensitive area coverage of $1.025 \times 0.850 \mathrm{~cm}^{2}$.
} 


\begin{tabular}{|c|c|c|}
\hline $\begin{array}{c}\text { Fluence } \\
\left(10^{12} \mathrm{p} / \mathrm{cm}^{2}\right)\end{array}$ & $A(\%)$ & $\begin{array}{c}C \\
\left(10^{-4} \mu \mathrm{m}^{4} \mathrm{~cm}^{-1}\right)\end{array}$ \\
\hline 0 & $83.13 \pm 0.04$ & $53.8 \pm 0.1$ \\
\hline 3.545 & $86.45 \pm 0.04$ & $54.5 \pm 0.1$ \\
\hline 7.045 & $91.55 \pm 0.04$ & $55.2 \pm 0.1$ \\
\hline 9.725 & $91.00 \pm 0.04$ & $55.8 \pm 0.1$ \\
\hline 19.445 & $86.18 \pm 0.04$ & $56.9 \pm 0.1$ \\
\hline 35.645 & $88.23 \pm 0.04$ & $54.6 \pm 0.1$ \\
\hline 51.525 & $85.22 \pm 0.04$ & $54.5 \pm 0.1$ \\
\hline
\end{tabular}

Table 1: Fluence in number of protons per $\mathrm{cm}^{2}$ traversing the central part of the aerogel sample. For each irradiation step, the fitted values of $A$ and $C$ are shown. The quoted errors are those determined by the fit.

\begin{tabular}{|c|c|c|}
\hline $\begin{array}{c}\text { Fluence } \\
\left(10^{12} \mathrm{n} / \mathrm{cm}^{2}\right)\end{array}$ & $A(\%)$ & $\begin{array}{c}C \\
\left(10^{-4} \mu \mathrm{m}^{4} \mathrm{~cm}^{-1}\right)\end{array}$ \\
\hline 0 & $81.47 \pm 0.04$ & $57.0 \pm 0.1$ \\
\hline 6.79 & $80.10 \pm 0.04$ & $62.3 \pm 0.1$ \\
\hline 9.95 & $79.26 \pm 0.04$ & $64.2 \pm 0.1$ \\
\hline 21.25 & $79.34 \pm 0.04$ & $64.7 \pm 0.1$ \\
\hline 38.35 & $83.72 \pm 0.04$ & $67.3 \pm 0.1$ \\
\hline 55.15 & $85.42 \pm 0.04$ & $68.3 \pm 0.1$ \\
\hline
\end{tabular}

Table 2: Fluence in units of $1 \mathrm{MeV}$ equivalent neutrons per $\mathrm{cm}^{2}$ absorbed by the aerogel sample irradiated with the mixed beam $\left(n, p, \pi^{ \pm}, \gamma\right)$. For each irradiation step, the fitted values of $A$ and $C$ are shown. The quoted errors are those determined by the fit.

\section{Behaviour with Irradiation}

In a RICH detector working at a $p p$ collider [10], the aerogel can be exposed to very intense irradiation. It is therefore important to check if this can result in a deterioration of the aerogel with the consequent degradation of the particle identification performances.

Inside the $\mathrm{LHCb}$ detector, the aerogel wall will be positioned at a radial distance of about $10 \mathrm{~cm} \mathrm{[10]} \mathrm{from} \mathrm{the} \mathrm{beam} \mathrm{and} \mathrm{at} \mathrm{about} \mathrm{one} \mathrm{metre} \mathrm{downstream} \mathrm{of} \mathrm{the} \mathrm{interaction}$ point. As a consequence, it will be exposed to a significant particle flux, up to $3.5 \times 10^{12}$ particles $/ \mathrm{cm}^{2}$ per year.

In order to investigate possible deterioration of the aerogel quality, samples of aerogel have been exposed to different sources of particles and their optical parameters monitored at different steps of the irradiation process.

\subsection{Sources of Irradiation}

One tile of dimensions $50 \times 50 \times 23 \mathrm{~mm}^{3}$ has been exposed to a proton source (IRRAD-1 [14]) using the CERN PS T7 East Hall beam. The primary proton beam had a momentum of $24 \mathrm{GeV} / \mathrm{c}$, the spot was $2 \times 2 \mathrm{~cm}^{2}$ wide with fluxes in the range $1-3 \times 10^{13} \mathrm{p} / \mathrm{cm}^{2} /$ hour. 


\begin{tabular}{|c|c|c|}
\hline $\begin{array}{c}\text { Dose } \\
(\mathbf{G y})\end{array}$ & $\boldsymbol{A}(\mathbf{\%})$ & $\begin{array}{c}\boldsymbol{C} \\
\mathbf{( 1 0}^{-\mathbf{4}} \boldsymbol{\mu m}^{\mathbf{4}} \mathbf{c m}^{\mathbf{- 1}} \mathbf{)}\end{array}$ \\
\hline 0 & $97.09 \pm 0.04$ & $44.4 \pm 0.1$ \\
\hline 38296 & $97.38 \pm 0.04$ & $44.0 \pm 0.1$ \\
\hline 73364 & $93.37 \pm 0.04$ & $39.0 \pm 0.1$ \\
\hline 115654 & $98.24 \pm 0.04$ & $41.5 \pm 0.1$ \\
\hline 229081 & $98.28 \pm 0.04$ & $41.0 \pm 0.1$ \\
\hline
\end{tabular}

Table 3: Dose in Gy absorbed by the hygroscopic aerogel sample irradiated with $\gamma$ rays from ${ }^{60} \mathrm{Co}$. For each irradiation step, the fitted values of $A$ and $C$ are shown. The quoted errors are those determined by the fit. The absorbed dose is the equivalent dose calculated for $\mathrm{SiO}_{2}$.

\begin{tabular}{|c|c|c|}
\hline $\begin{array}{c}\text { Dose } \\
(\mathbf{G y})\end{array}$ & $\boldsymbol{A}(\mathbf{\%})$ & $\begin{array}{c}\boldsymbol{C} \\
\mathbf{( 1 0}^{-\mathbf{4}} \boldsymbol{\mu m}^{\mathbf{4}} \mathbf{c m}^{\mathbf{- 1}} \mathbf{)}\end{array}$ \\
\hline 0 & $94.33 \pm 0.3$ & $78.9 \pm 0.2$ \\
\hline 37305 & $96.59 \pm 0.3$ & $77.0 \pm 0.2$ \\
\hline 72373 & $98.18 \pm 0.5$ & $61.3 \pm 0.2$ \\
\hline 114663 & $97.99 \pm 0.5$ & $79.4 \pm 0.2$ \\
\hline 228090 & $98.36 \pm 0.3$ & $71.0 \pm 0.2$ \\
\hline
\end{tabular}

Table 4: Dose in Gy absorbed by the hydrophobic aerogel sample irradiated with $\gamma$ rays. For each irradiation step, the fitted values of $A$ and $C$ are shown. The quoted errors are those determined by the fit. The absorbed dose is the equivalent dose calculated for $\mathrm{SiO}_{2}$.

Because of the small size of the beam, irradiation was concentrated only in the center of the tile.

The exposure to IRRAD-1 was made in six steps: Table 1 summarises the fluence accumulated at each step. The total fluence amounts to $51.525 \times 10^{12}$ protons $/ \mathrm{cm}^{2}$. The first three irradiation steps correspond each to about one year of operation of the aerogel $\mathrm{RICH}$ of $\mathrm{LHCb}$ at $\mathrm{LHC}$, the last ones to larger doses that will not probably be reached in the lifetime of the detector.

Another tile of aerogel was exposed to neutrons, this time irradiating uniformly the whole tile. Neutrons were obtained using the CERN PS T8 proton beam (with momentum of $24 \mathrm{GeV} / \mathrm{c}$ ) interacting on lead and carbon targets (IRRAD-2 [15]). Secondary particles in the irradiation cavity were neutrons (energy range: $50 \mathrm{KeV}-1 \mathrm{MeV}$ ), p, $\pi^{+}, \pi^{-}$(energy range: $0.3-4 \mathrm{GeV}$ ) and $\gamma$ (energy range: $100 \mathrm{KeV}-100 \mathrm{MeV}$ ). An energy cut on the spectrum (energy higher than $100 \mathrm{KeV}$ ) was applied to calculate the dose of neutrons. With this selection, $1 \mathrm{MeV}$ equivalent neutron flux is $5.59 \times 10^{11} \mathrm{n} / \mathrm{cm}^{2} /$ hour at $50 \mathrm{~cm}$ from the flux axis, $1.11 \times 10^{12} \mathrm{n} / \mathrm{cm}^{2} /$ hour at $10 \mathrm{~cm}$. The total accumulated fluence was $55.15 \times 10^{12} \mathrm{n} / \mathrm{cm}^{2}$, corresponding approximately to about 15 years of data taking of LHCb. Table 2 summarises the measurements at each irradiation step. 
a)
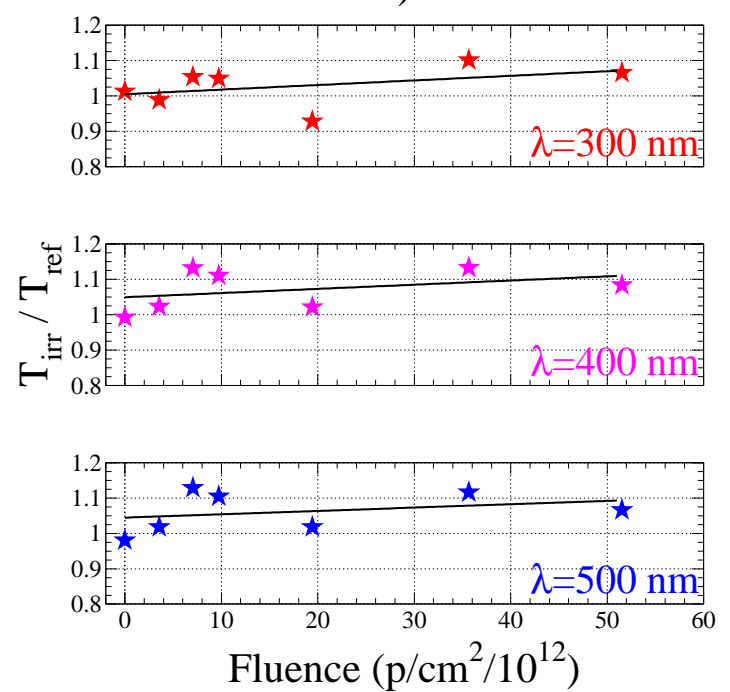

b)
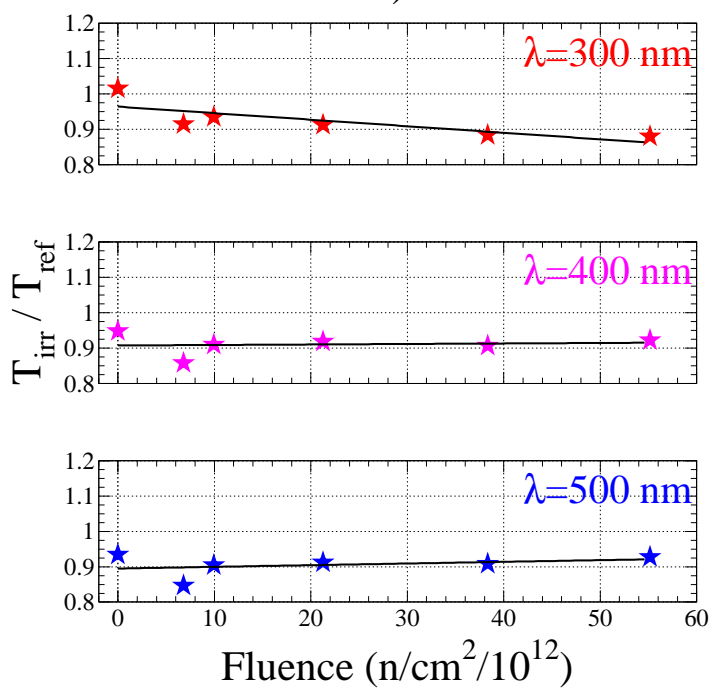

Figure 4: Transmittance ratios $T_{i r r} / T_{\text {ref }}$ as a function of the absorbed dose at different wavelengths with a) protons and b) neutrons. A linear fit has been superimposed.

a)

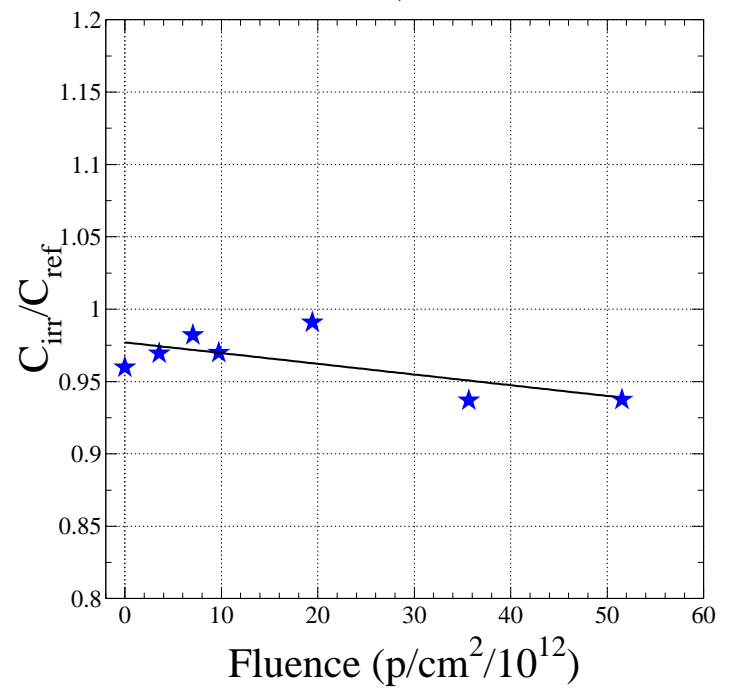

b)

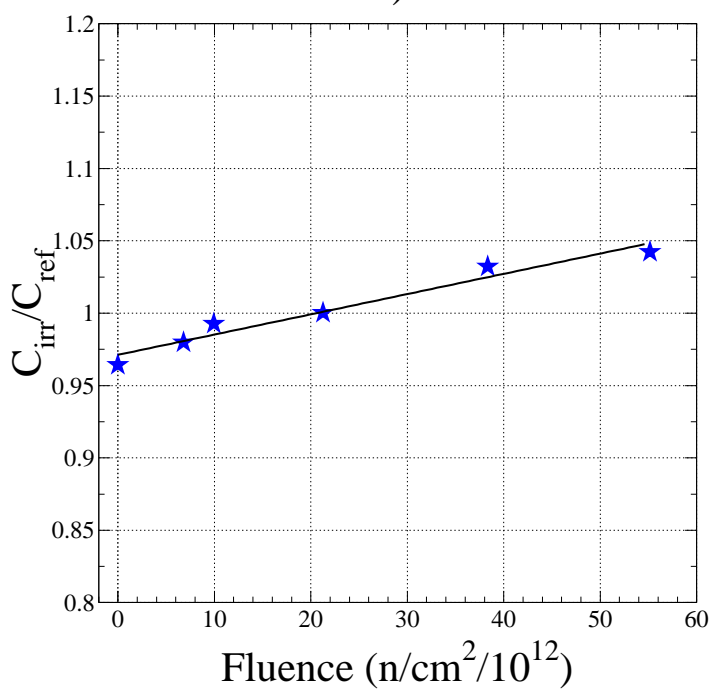

Figure 5: Clarity ratios $C_{i r r} / C_{\text {ref }}$ as a function of the absorbed dose with a) protons and b) neutrons. A linear fit has been superimposed. 
a)

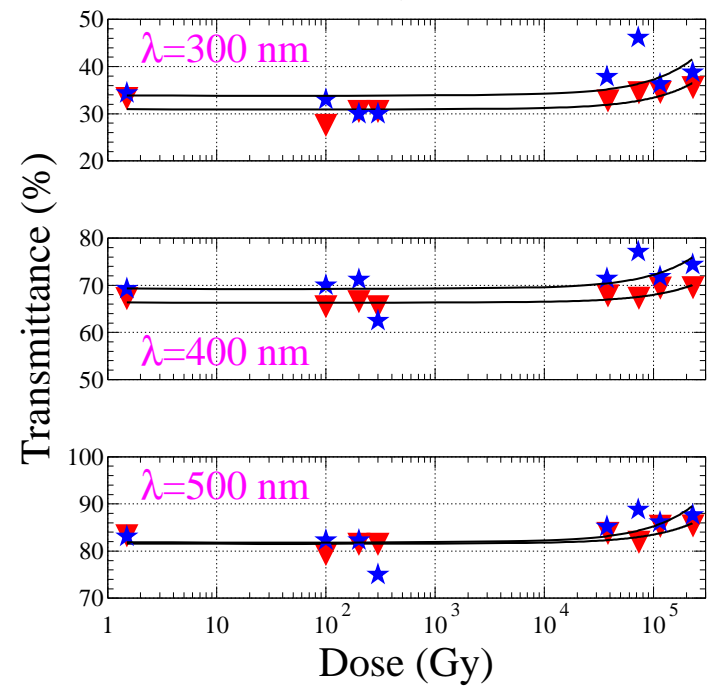

b)

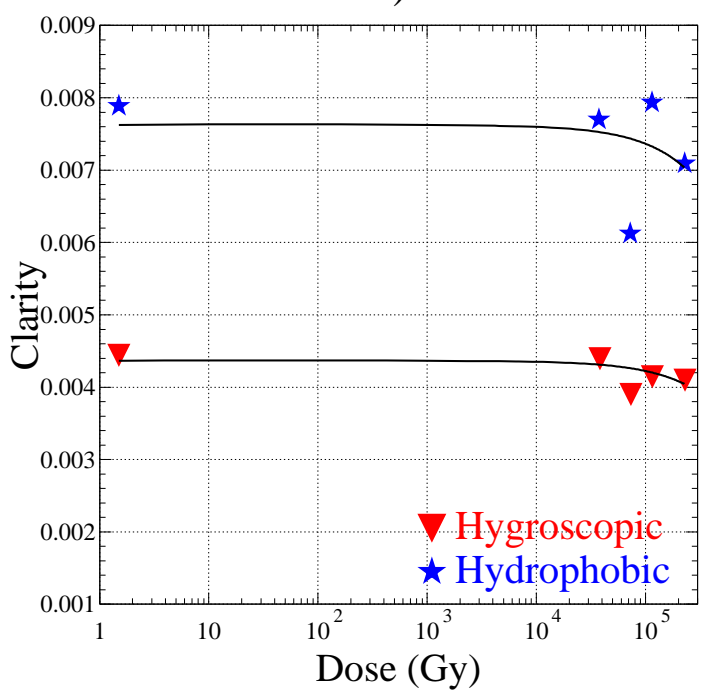

Figure 6: a) transmittance as a function of the absorbed dose of $\gamma$ at different wavelengths; b) clarity as a function of the absorbed dose. A linear fit has been superimposed.

Finally, tiles of hygroscopic and hydrophobic aerogel were irradiated with $\gamma$ from a radioactive ${ }^{60} \mathrm{Co}$ source used in a Gammacell ${ }^{2} 220$ unit [16] located at the "Istituto Superiore di Sanità" in Rome. The source provides an uniform irradiation by gamma rays with an energy of 1.3 and $1.7 \mathrm{MeV}$. Also in this case, the transmittance was monitored as an ageing indicator after every irradiation period. Five irradiation steps provided a total dose of $\simeq 230 \mathrm{kGy}$, corresponding to the dose absorbed by the innermost region of the aerogel radiator in $\sim 30$ years of operation of LHCb. Table 3 summarises the measurements with the hygroscopic aerogel, and Table 4 those concerning the hydrophobic aerogel. The results with $\gamma$ irradiation on hydrophobic aerogel agrees with previous tests [17].

\subsection{Measurements and Results}

The transmittance of the irradiated tiles was measured before and after each irradiation step, and the curves were then fitted with the Hunt equation (1) to determine the $A$ and $C$ parameters. During irradiation, the hygroscopic tiles were sealed in a polyethylene bag filled with nitrogen to ensure isolation from humid air. Each time the transmittance was measured, the aerogel block was exposed to air for about one hour.

In order to separate effects due to the handling of the tiles, measurements were made systematically on a reference tile, with optical parameters $A$ and $C$ very close to those of the irradiated ones. The comparison of the two samples was then made to account for the effects due to handling in the irradiation measurements. The ratio of the transmittances of the irradiated and the reference samples is plotted in Fig. 4 for different wavelengths as a function of the absorbed dose. Fig. 5 presents the ratios for the clarity. For proton irradiation, no evidence of degradation of the aerogel optical parameters is visible: both

\footnotetext{
${ }^{2}$ Gammacell $_{\circledast} 220$ is a brand name of the Atomic Energy of Canada Limited (AECL) and MDS Nordion International.
} 


\begin{tabular}{|c|c|c|}
\hline Absorbed dose (Gy) & Hygroscopic & Hydrophobic \\
\hline 0 & $1.0336 \pm 0.0003$ & $1.0297 \pm 0.0003$ \\
18797.0 & $1.0326 \pm 0.0003$ & - \\
19788.0 & - & $1.0304 \pm 0.0003$ \\
\hline
\end{tabular}

Table 5: Index of refraction $n$ for hygroscopic and hydrophobic aerogel samples as a function of the absorbed dose of $\gamma$ at $\lambda=632.8 \mathrm{~nm}$. Errors are described in Section 4.2.

parameters $A$ and $C$ are independent of the fluence.

The results from the neutron exposure show a more complex response. While $T$ does not change for $\lambda$ above $400 \mathrm{~nm}$, the value of the clarity factor $C$ has an increasing trend, as if the structure of the silica aggregates and empty bubbles inside the tile were modified by the high flux of particles traversing the aerogel sample. The worsening in the clarity factor is mainly determined by the behaviour at small values of wavelength as can be seen from the transmittance ratios in Fig. $4 \mathrm{~b}$.

No detectable decrease of the aerogel optical quality has been found under gamma irradiation, as shown in Fig. 6: the transmittance $T$ is unchanged within the experimental uncertainties. For this kind of irradiation, the index of refraction was measured in the initial conditions and after $\simeq 20000 \mathrm{~Gy}$. Also for this parameter no detectable variations have been observed, as shown in Table 5. No comparison with reference tiles was available in the case of $\gamma$ irradiation.

\section{Behaviour with Humidity}

Hygroscopic aerogel can absorb water vapour, forming $\mathrm{OH}$ groups. These can be eliminated by baking the aerogel at high temperatures. The humidity test was performed using the following procedure. The aerogel tile was baked at a temperature of $\simeq 500^{\circ} \mathrm{C}$ for about four hours. That temperature was reached in about five hours in order to avoid possible cracks due to thermal stress. At the end of this first baking process, the index of refraction and transmittance were measured and $A$ and $C$ were determined.

The aerogel block was then exposed to humid air for about one week; the optical parameters were remeasured. Finally, a new baking cycle was done in order to find out if it was possible to restore the initial values.

\subsection{The Experimental Set-up}

The setup used to expose aerogel to humid air was similar to a greenhouse. Fig. 7 shows the set-up: the aerogel tile was placed on a high precision balance. Glasses of water were placed near the tile, inside the transparent box, creating a humid environment. The temperature of the room was about $24^{\circ} \mathrm{C}$, and the variations of the aerogel weight caused by the absorption of water were recorded by the balance as a function of temperature and of relative humidity which were monitored with a computer and are shown in Fig. 8. This figure shows also the variation of the aerogel weight as a function of time. The steep rise indicates that at the beginning the aerogel can absorb a large amount of water vapour, then the absorption of water slows down. Oscillations are due to a day/night effect in the temperature of the room. 


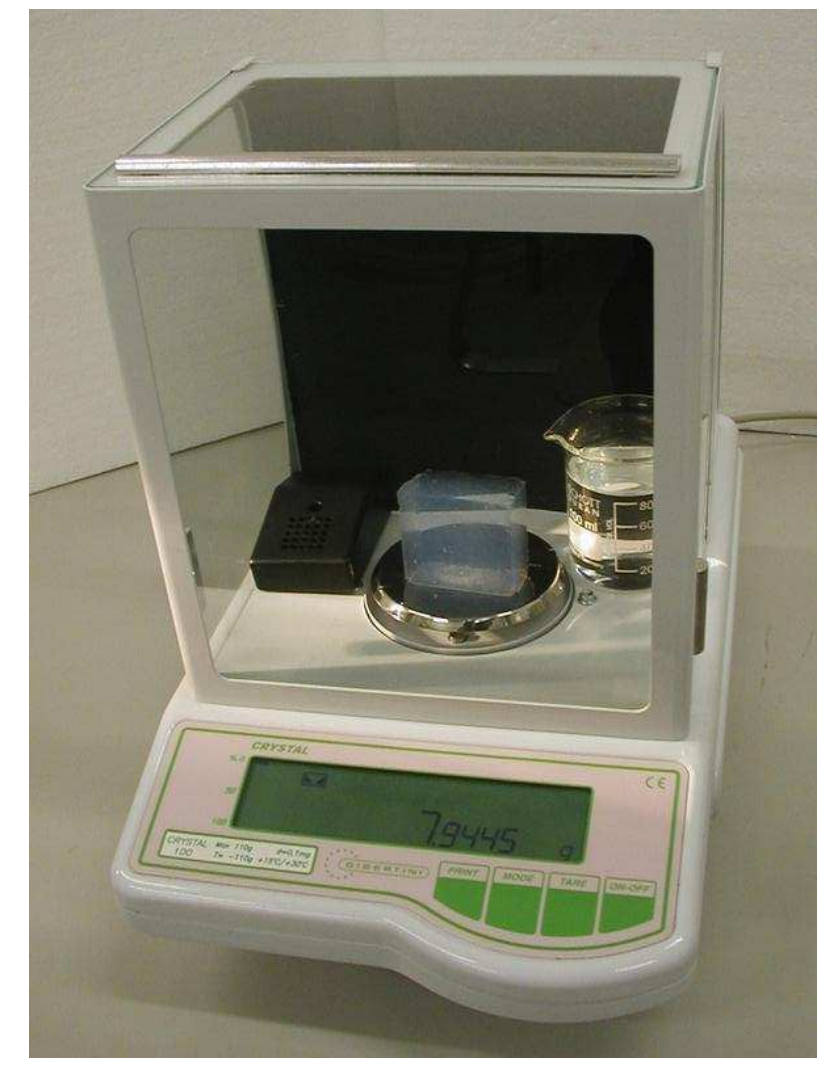

Figure 7: The experimental set-up used for the humidity test. The aerogel is located on the plate of the balance, the atmosphere inside is saturated with vapour of water.

The index of refraction $n$ and light transmission $T$ of the hygroscopic aerogel were measured as a function of the humidity absorbed by the tile. If the relative humidity of the room (where measurements were done) is different from the one of the aerogel environment inside the box, the aerogel can absorb or expel some water to reach equilibrium. This is a quite fast process, so in order to keep the aerogel under uniform conditions and minimize variations of weight, measurements were made turning off the air conditioning system of the room, inducing a relative humidity between $60 \%$ and $70 \%$.

\subsection{Measurements of the Refractive Index}

The prism method, as described in Section 2.2, provides an accurate measurement of $n$. The uncertainty on $n$ is related to that of the two angles $\Phi$ and $\theta_{\text {out }}$; this latter is determined by the two distances $x$ and $L$, as shown in Fig. 3. From formula (2), the error on the refractive index is given by:

$$
\sigma_{n}^{2}=\left(\frac{\partial n}{\partial \Phi}\right)^{2} \sigma_{\Phi}^{2}+\left(\frac{\partial n}{\partial x}\right)^{2}\left[\sigma_{x}^{2}+\frac{x^{2}}{L^{2}} \sigma_{L}^{2}\right]
$$

The laser beam spot on the CCD display presents a central region about $2 \mathrm{~mm}$ wide with halos around it, as shown in Fig. 9. The center of gravity of the image was used to 

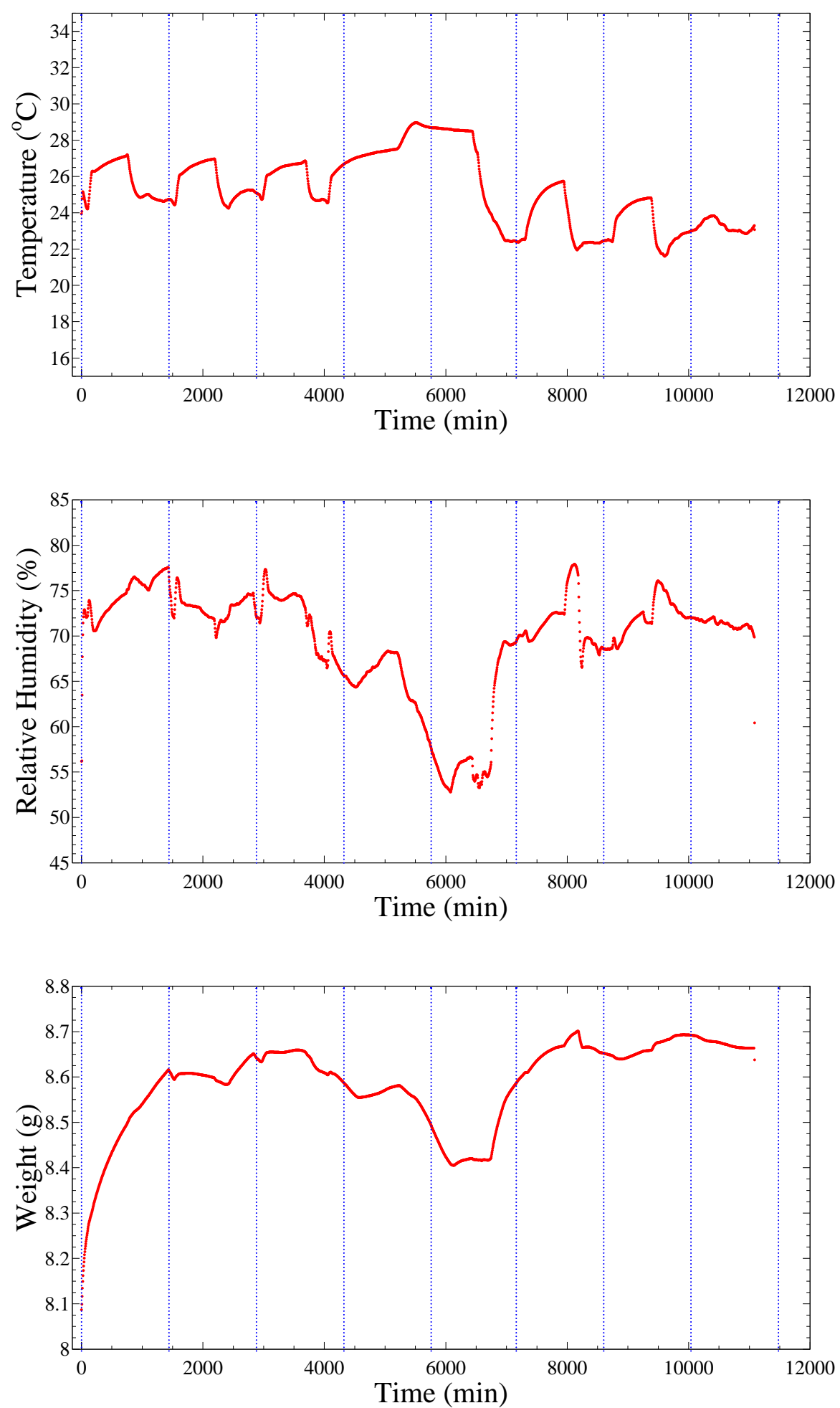

Figure 8: Temperature $\left({ }^{\circ} \mathrm{C}\right)$ and relative humidity $(\%)$ of the room and weight $(\mathrm{g})$ variation of the aerogel tile as a function of time (in minutes); vertical lines indicate intervals of 24 hours. 


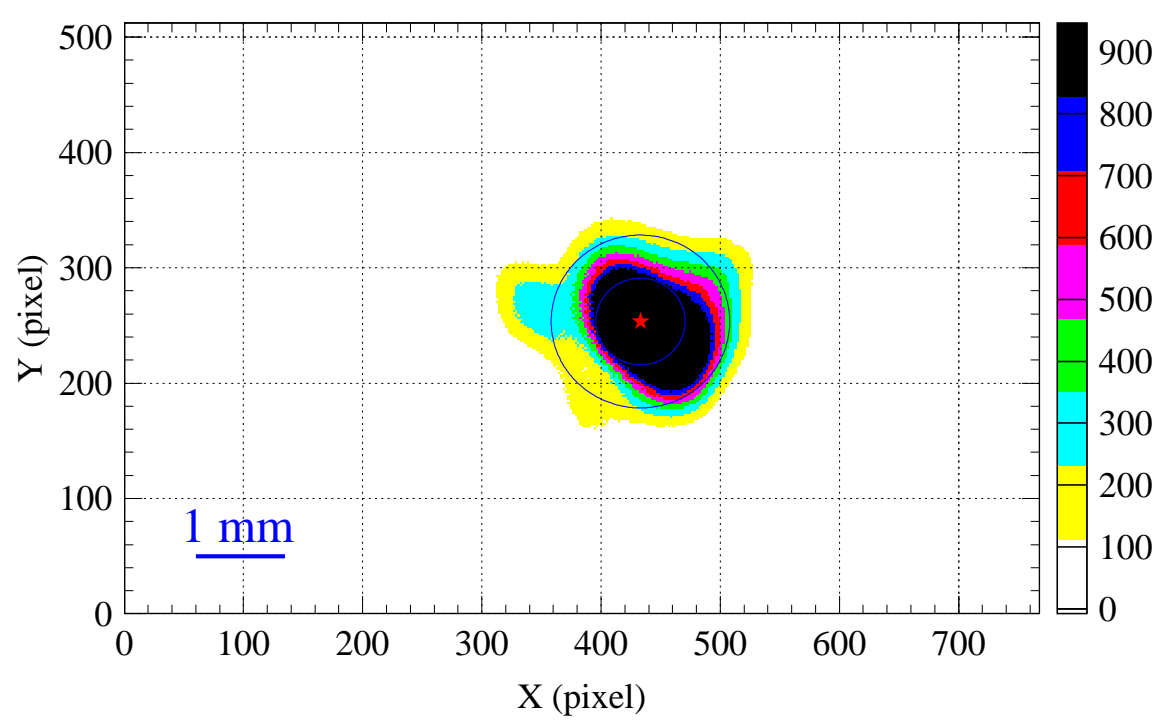

Figure 9: Typical picture of the laser beam spot from the CCD. The star indicates the center of gravity of the image; $1 \mathrm{~mm}$ and $2 \mathrm{~mm}$ diameters circles are also plotted.

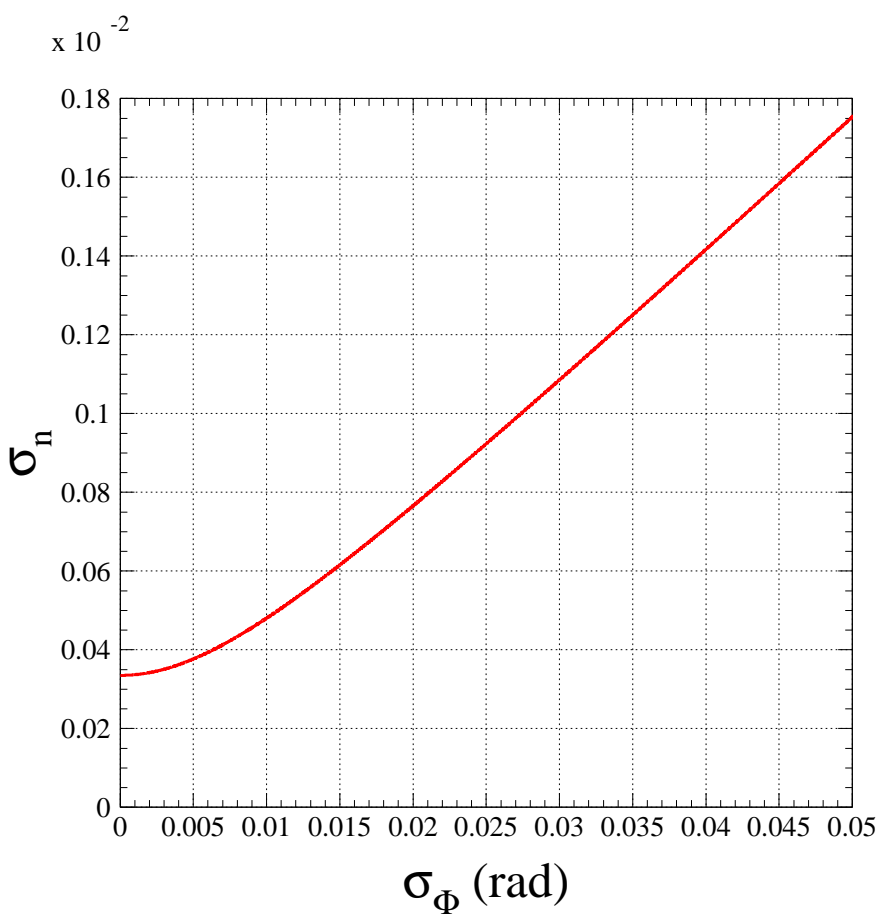

Figure 10: The error $\sigma_{n}$ as a function of the uncertainty on the angle $\Phi$ at the tile vertex when $\sigma_{x}=0.5 \mathrm{~mm}$ and $\sigma_{L}=5 \mathrm{~mm}$. 


\begin{tabular}{|c|c|c|c|c|}
\hline $\boldsymbol{m}$ & $8.06 \mathrm{~g}$ & $8.09 \mathrm{~g}$ & $8.35 \mathrm{~g}$ & $8.63 \mathrm{~g}$ \\
\hline $\boldsymbol{n}$ & 1.0335 & 1.0331 & 1.0345 & 1.0363 \\
$\boldsymbol{\delta} \boldsymbol{n}$ & 0.0005 & 0.0009 & 0.0012 & 0.0029 \\
\hline
\end{tabular}

Table 6: Index of refraction $n$ measured with the green laser source as a function of weight. Spreads in the measurements are also shown.

\begin{tabular}{|c|c|c|c|c|}
\hline $\boldsymbol{m}$ & $8.06 \mathrm{~g}$ & $8.07 \mathrm{~g}$ & $8.35 \mathrm{~g}$ & $8.61 \mathrm{~g}$ \\
\hline $\boldsymbol{A} \mathbf{( \% )}$ & $82.49 \pm 0.04$ & $81.94 \pm 0.04$ & $82.54 \pm 0.04$ & $81.35 \pm 0.04$ \\
$\boldsymbol{C}\left(\mathbf{1 0}^{-\mathbf{4}} \boldsymbol{\mu m}^{\mathbf{4}} \mathbf{c m}^{\mathbf{- 1}}\right)$ & $55.1 \pm 0.1$ & $57.3 \pm 0.1$ & $61.9 \pm 0.1$ & $69.1 \pm 0.1$ \\
\hline
\end{tabular}

Table 7: Values of the parameters $A$ and $C$ as a function of weigth for different steps in the humidity test.

measure the $x$ displacement on the screen. The precisions on $x$ and $L$ are $\sigma_{x} \cong 0.5 \mathrm{~mm}$ and $\sigma_{L} \cong 5 \mathrm{~mm}$ respectively. The uncertainty on the angle $\Phi$ is essentially determined by the small deviation from $\Phi=\pi / 2$ due to local imperfections on the surfaces of the tiles crossed by the laser beam. Assuming $\sigma_{\Phi}=0$, the uncertainty on $n$ is $\sigma_{n} \cong 0.0003$. The contribution to $\sigma_{n}$ due to the uncertainty on the angle $\Phi$ is plotted in Fig. 10 . The contribution to $\sigma_{n}$ due to the definition of the minimum displacement condition is negligible $\left(\sigma_{n}<7 \cdot 10^{-5}\right)$.

The refractive index was then determined from several measurements corresponding to different entrance points on the aerogel. The mean value was taken as the final measurement.

Table 6 lists the values of $n$ as a function of the absorbed humidity reflected in the increase of the aerogel weight. The spreads $\delta n$ (R.M.S.) of each set of measurements are also shown. These spreads are related to local non-homogeneities of the tiles, but they are also connected to the fast variation of the aerogel weight during the measurement procedure (as explained in Section 4.1). The refraction index $n$ increases with the amount of absorbed water vapour. The maximum variation of $\Delta n / n$ (corresponding to an increase of mass of $6.7 \%$ ) was $0.3 \%$ for the green laser source. Such a kind of variation would give a large contribution to the performances of particle identification with RICH detectors.

\subsection{Measurement of Transmittance}

Fig. 11 shows the results for transmittance $T$ at different wavelengths and for the clarity factor $C$ measured at different steps of water absorption. A linear fit has been superimposed. For $C$ an increase as a function of the absorbed humidity is measured in the data. Table 7 summarizes the results of the measurements.

It must be noted that the aerogel tiles of the LHCb RICH detector will be encapsulated in an hermetic box, and therefore the exposure to humidity will be certainly lower than the one considered for this test. 
a)

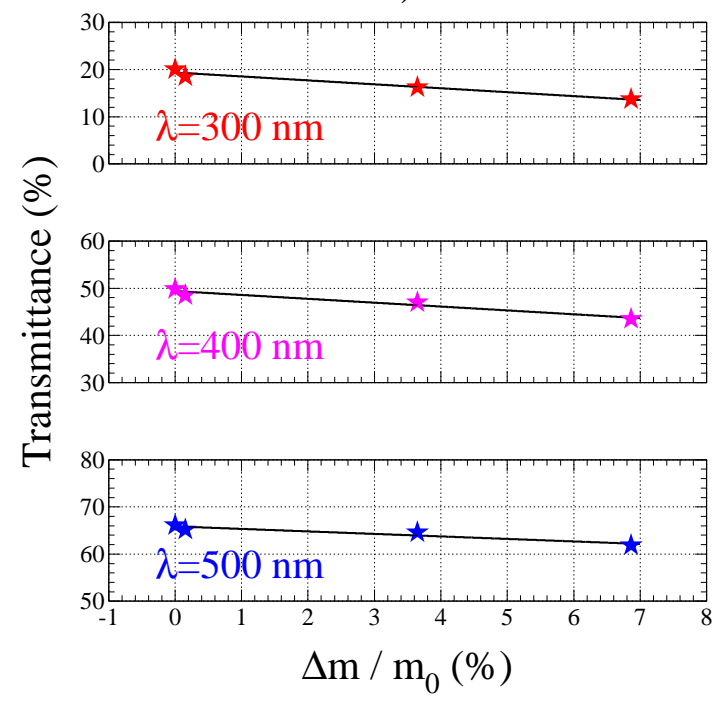

b)

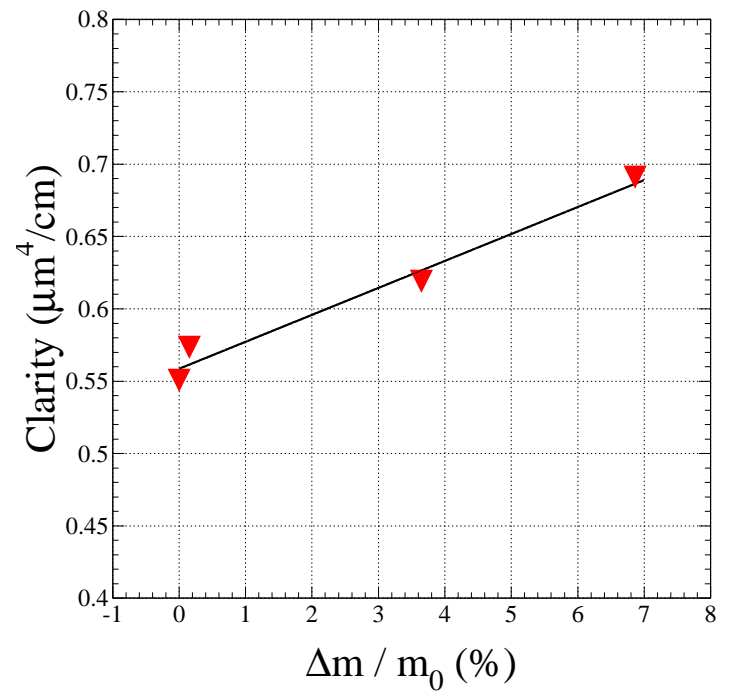

Figure 11: a) transmittance as a function of $\Delta m / m_{0}(\%)$ at different wavelengths during the humidity test; b) clarity as a function of $\Delta m / m_{0}(\%)$.

\section{Conclusions}

Ageing effects on aerogel due to irradiation with different types of incident particles and due to humidity absorption were investigated by monitoring the optical parameters characterising the quality of the aerogel tiles. The parameter $A$, the clarity factor $C$ and the index of refraction $n$ were measured.

The performed measurements show no evidence of ageing of the aerogel when irradiated with protons and $\gamma$, and a moderate degradation of the clarity when irradiated with neutrons. On the other hand, the humidity test revealed that a prolonged exposure to humid air does not irreversibly degrade the properties of the aerogel, which are completely restored by baking the exposed sample at $\simeq 500^{\circ} \mathrm{C}$.

\section{Acknowledgements}

We thank A. R. Buzykaev, E. A. Kravchenko and A. P. Onuchin of the Budker Institute of Nuclear Physics and A. F. Danilyuk of the Boreskov Institute of Catalysis, Novosibirsk who provided the aerogel samples used for these ageing tests. We are grateful to $\mathrm{M}$. Monteleone of the "Istituto Superiore di Sanità" and to Dr. C. Bosio of the University of Rome "La Sapienza" for their help with the ${ }^{60}$ Co source. We acknowledge also the TA1-SD section and particularly M. Glaser for the proton and neutron irradiation. We are thankful to E. Sibilia from the Department of Material Science of the Milano Bicocca University for the availability of the oven, and to N. Ludwig from the Physics Department of the Milano University for kindly providing the first set-up for the humidity absorption. We acknowledge the collaboration of C. Rovelli during her summer studentship at CERN. 


\section{References}

[1] M. Cantin et al., Nucl. Instr. and Meth., A118 (1974), 177.

[2] A. F. Danilyuk et al., Nucl. Instr. and Meth., A433 (1999), 406.

[3] H. Yokogawa and M.Yokoyama, J. Non-Cryst. Solids 186 (1995) 23.

[4] R. De Leo et al., Nucl. Instr. and Meth., A401 (1997), 187.

[5] A. K. Gougas et al., Nucl. Instr. and Meth., A421 (1999), 249.

[6] A. R. Buzykaev et al., Nucl. Instr. and Meth., A433 (1999), 396.

[7] R. De Leo et al., Nucl. Instr. and Meth., A457 (2001), 52.

[8] M. Y. Barnykov et al., Nucl. Instr. and Meth., A419 (1998), 584.

[9] HERMES Coll., Nucl. Instr. and Meth., A417 (1998), 230.

[10] LHCb Coll., LHCb RICH TDR, CERN/LHCC/2000-0037.

[11] R. Battiston et al., Nucl. Instr. and Meth., A409 (1998), 458.

[12] T. Matsumoto et al., physics/0309032 Sep. 2003

[13] A. J. Hunt et al., Mat. Res. Soc. Symp. Proc. (1984) 275.

[14] http://irradiation.web.cern.ch/irradiation/irrad1.htm

[15] http://irradiation.web.cern.ch/irradiation/irrad2.htm

[16] http://www.mds.nordion.com/

[17] S. K. Sahu et al., Nucl. Instr. and Meth., A382 (1996), 441. 